\title{
A design-driven exploration of photovoltaic applications in electric mobility systems
}

\author{
Alonzo Sierra \\ Faculty of Engineering Technology \\ University of Twente \\ Enschede, The Netherlands \\ a.sierrarodriguez@utwente.nl
}

\author{
Angèle Reinders \\ Faculty of Engineering Technology \\ University of Twente \\ Enschede, The Netherlands \\ a.h.m.e.reinders@utwente.nl
}

\begin{abstract}
This paper presents the results of a conceptual design study regarding photovoltaic applications in electric mobility systems while considering technical, user, regulatory and aesthetic aspects. A total of eleven different design projects were developed, mostly encompassing innovative applications for $\mathrm{EV}$ charging infrastructure, vehicle-integrated PV systems, or a combination of both. Four of these conceptual designs are presented and evaluated in detail in this paper, showing the potential advantages that PV technology could bring to electric mobility systems not just in terms of $\mathrm{CO}_{2}$ emissions reduction but also regarding product aesthetics and user experience.
\end{abstract}

Keywords-PV systems, PV for transport, design, electric vehicles, charging.

\section{INTRODUCTION}

The current uptake in the use of electric vehicles (EV) will lead to a substantial reduction of global $\mathrm{CO}_{2}$ emissions, since road vehicles currently account for around $70 \%$ of greenhouse gas emissions in the transport sector [1]. This reduction, however, greatly depends on the carbon intensity of electricity used for charging EVs. For instance, in the case of grids with a high share of fossil fuel plants, an EV's $\mathrm{CO}_{2}$ emissions per kilometer driven could be relatively high [2][3] . In order to reduce these emissions it is of particular interest to charge EVs with renewable energy technologies such as solar photovoltaics (PV).

Since there is at present limited experience with the application of PV systems in electric vehicles and/or their charging infrastructure, a conceptual design study was carried out in 2019 with students of the Studies of Industrial Design Engineering and Mechanical Engineering at the University of Twente (UT) in the Netherlands to explore how innovative PV applications for these technologies could look like in a nearfuture mobility context. This paper presents the results of this design study consisting of several innovative conceptual designs for the integration of PV in future electric mobility systems which are critically evaluated regarding their expected benefits, points of improvement and barriers towards implementation in practice. The design study and the evaluation of its results have an interdisciplinary scope by considering technical, user, regulatory and aesthetic aspects. This fits to existing theoretical frameworks for designing with photovoltaics [4][5] which have been developed by means of the execution of actual design projects in the past 15 years at the University of Twente [6-9].
This paper is structured as follows: Section II provides an introduction to the context of the design project, with Section III showcasing four of the resulting designs and their evaluation. Finally, Section IV will discuss several key findings and provide some conclusions to this study.

\section{Project CONTEXT}

The design project presented in this paper was executed in the 2019 course 'Sources of Innovation', which is targeted at developing novel designs using innovative sustainable technologies. Thirty-three master students from the Master programmes of Industrial Design Engineering and Mechanical Engineering participated in this course, in which they were tasked with a conceptual design brief aimed at the development of a "product-service combination for solar PV powered EV charging". In the course the conventional product development process is accelerated by applying several innovation methodologies [4][10-12] such as the ideation-enhancing Innovation Phase Model (IPM), the Russian technical problem solving method TRIZ and Platform-driven Product Development (PDPD) which is commonly applied in the global product industry, among others.

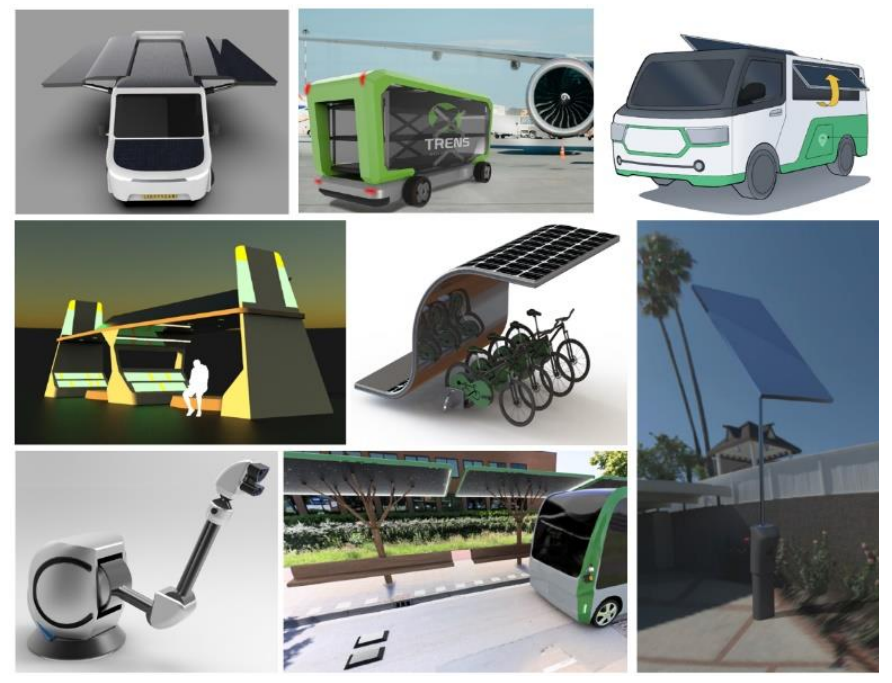

Fig. 1. Examples of conceptual designs developed by students during the 2019 'Sources of Innovation' course at the University of Twente . From left to right, top to bottom: Lightyear Bus, Solar Luggage Vehicle, Zun solar van, Solar Bus Terminal, Lock n'Load e-bike shed, Solarshare+ PV charge point, Robotic EV charger and Solar Train Stop. 
In the past this approach has been successfully applied in other projects that aimed at designing with concentrator photovoltaics, luminescent solar concentrators, smart grid technologies and other PV applications [8][9][13][14]. A significant part of the resulting conceptual designs of the course Sources of Innovation usually has an innovative, but applicable, realistic scope.

To provide a realistic context and to stimulate ideation, during the assignment, students were asked to imagine being part of the design team of one of three companies currently developing PV applications for electric mobility: Lightyear [15], IM Efficiency [16] and Trens Solar Trains [17]. The conceptual design was required to consider technical aspects, user interaction, estimated costs, regulatory framework, and product aesthetics. In addition to this, the concept had to be suitable for the Netherlands as well as other international locations of the students' choice such as China, India and the United States.

\section{DESIGN RESUlTS}

The results of the student design projects covered a wide range of application purposes and vehicle types; designs mostly focused on either charging infrastructure or on vehicleintegrated PV systems, with some offering product-service combinations or service-only solutions. Developed concepts included a van with movable PV side panels, a robotic EV charger and a bike shed with integrated PV cells, among others (see Fig. 1).

This section will showcase four examples of the designs developed by the student teams: the first two concepts focus on PV-powered charging infrastructure while the other two address vehicle-integrated PV applications. Each concept will be introduced as it was conceived by the designers, followed by an evaluation on each design's limitations and possible improvements that could be explored in the future.

\section{A. Solar Bus Terminal}

This design concept consists of a structure serving as a terminal for electric city buses where a PV system located on top of the structure charges battery packs which are then used for recharging the buses while they are parked at the terminal (see Fig. 2). The battery packs are located in a series of slots at the

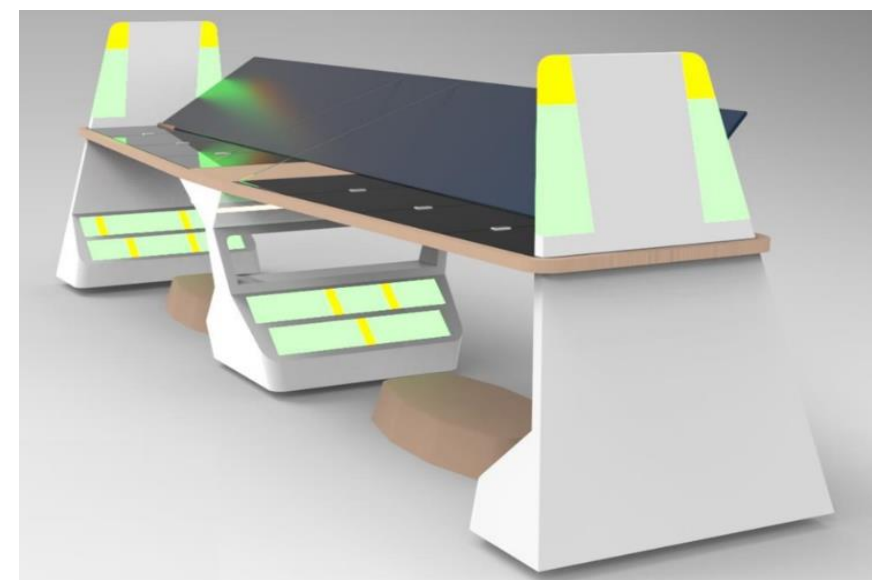

Fig. 2. Solar bus terminal concept by M. Dijkstra, A. Suresh and S. V. Ramana. base of the structure where they can be conveniently accessed by bus drivers who will then manually swap a depleted battery pack for a charged one to quickly recharge their vehicle. The state of charge is visually indicated by a series of lights in the upper section of the battery pack so that drivers can identify which pack they need to replace.

The terminal is fitted with LED lighting in the two pillars located next to the PV system as well as in several displays near the passenger waiting area. The LEDs can show yellow or green colouring, and the ratio between these two colours is used to indicate how many battery packs have been charged by the PV system at any given moment. This visual feedback, together with a design aesthetic which highlights the PV modules on top of the structure, is intended to clearly communicate the sustainabilityoriented focus of the bus service to its end users.

Design Evaluation - One of the main limitations of the current design involves the difficulties presented by the proposed charging process which requires drivers to exit the bus for a brief period of time in order to replace the battery packs. This could be problematic during peak traffic hours or at times with less favourable weather conditions, and might also become an inconvenience to passengers as additional waiting time could be required. In addition to this, the suggested capacity for the battery packs (around $20 \mathrm{kWh}$ ) would make them too heavy to be carried by a single person and while it is possible to use a larger number of small battery packs instead of a single large pack, this would only increase the required time for swapping even further. It is possible to use automation to speed up the swapping process, but this has already proven to be difficult to achieve in practice [18].

An additional limitation from the presented design is the potential self-shading of the PV system due to the pillars located at its sides. This issue can be solved by designing less intrusive pillars or removing them altogether. Additional PV cells could also be integrated into other parts of the structure but this would depend on the specific location and orientation of the terminal structure.

\section{B. Solar Train Stop}

In this concept, a small PV system is integrated with a street bench in order to create a stop for electric city trains which provides shelter to users while also acting as a local source of renewable energy (see Fig. 3). Vehicle charging is done through a set of charging pads located on the street: these pads use induction charging to provide a small amount of charge to the vehicle while it stops to pick up and drop off passengers. Adequate contact between the train and the charging pads is ensured through a system of sensors and pneumatic actuators which uses compressed air and small heaters to clear the pad surface in case of rain or snow. The pads are only active when the vehicle is above them in order to prevent safety risks to other vehicles or pedestrians.

The train stop design is intended to provide a comfortable environment for waiting passengers while making the vehicle charging process as unobtrusive as possible from both the driver and the user's perspective. The station's visual aesthetics replicate the shape of a tree in order to help it blend in with the urban environment as well as providing a more pleasant and eco- 


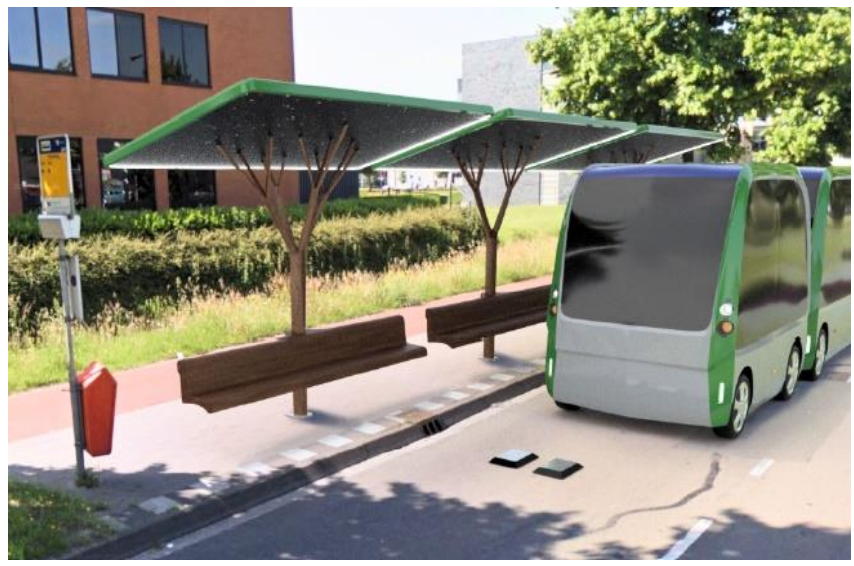

Fig. 3. Solar train stop concept by S. Elango, U. Parvangada and G. Ribeiro.

friendly appearance to users. This distinct look is also important for making the station recognisable as part of a sustainable transportation system. The concept also has a modular design since the number of benches can be modified depending on the available space and the required PV capacity.

Design Evaluation - During its operation, the solar train will only be in front of the station for several seconds while passengers board or exit the vehicle. This means that a high charging speed is required and, while induction pads are already able to provide ultra-fast charging with speeds of up to $200 \mathrm{~kW}$ [19], it is unclear if the PV system can directly provide a sufficient amount of instantaneous power which would also put a significant strain on the local grid. Nevertheless, even if this charging system is only able to provide a small fraction of the vehicle's energy requirements the design could allow for smaller on-board batteries to be used, reducing the solar train's weight and increasing its energy efficiency.

As was the case with the Solar Bus Terminal concept introduced in the previous subsection, this concept could be adapted for charging other types of vehicles such as EVs or electric bicycles in parking lots. Additionally, similar designs applied to other objects commonly found in the urban environment such as street signs and lamps could be developed for charging parked vehicles.

\section{Lightyear Bus}

This concept aims to reduce range anxiety among EV users by creating an electric van with integrated PV panels which functions as a mobile charging unit (MCU) used for road assistance. The vehicle has a large PV array on the roof as well as additional cells integrated to the front, sides and back; the side panels can be rotated and extended to nearly double the total area of the array when the MCU is not in motion (see Fig. 4).

PV power generated by the MCU is stored in a battery bank which is then used for charging EVs through a series of charging ports located on the sides of the vehicle. The size of the MCU is similar to that of a small bus which provides enough space for charging up to four EVs at the same time.

EV users can summon an MCU to their location whenever they need to charge their vehicle which is particularly useful in areas with limited charging infrastructure; this on-demand service is requested though a mobile application which allocates the closest available MCU to each user. If several users in the same area request the charging service, it is possible to dispatch the MCU to a central location which is accessible to all users who need it.

Design Evaluation - In order to maximise the size of its PV system the MCU was designed to have large dimensions, particularly lengthwise. This design choice could make the vehicle too difficult to move around in practice, meaning that future designs need to consider the trade-off between vehicle size and the desired PV capacity. A similar issue is likely to arise with the extendable side panels which need free space around the MCU to be deployed.

As was the case with the concepts involving EV charging stations, it is unclear whether the PV system can provide a sufficient amount of instantaneous power for its intended use. The required energy could be produced at a different time and stored in the MCU's battery bank but a large storage capacity would be required for powering both several user EVs in addition to the MCU itself. This might not be feasible in practice since there is an upper limit for on-board storage capacity due to the trade-off between a vehicle's weight and its energy efficiency.
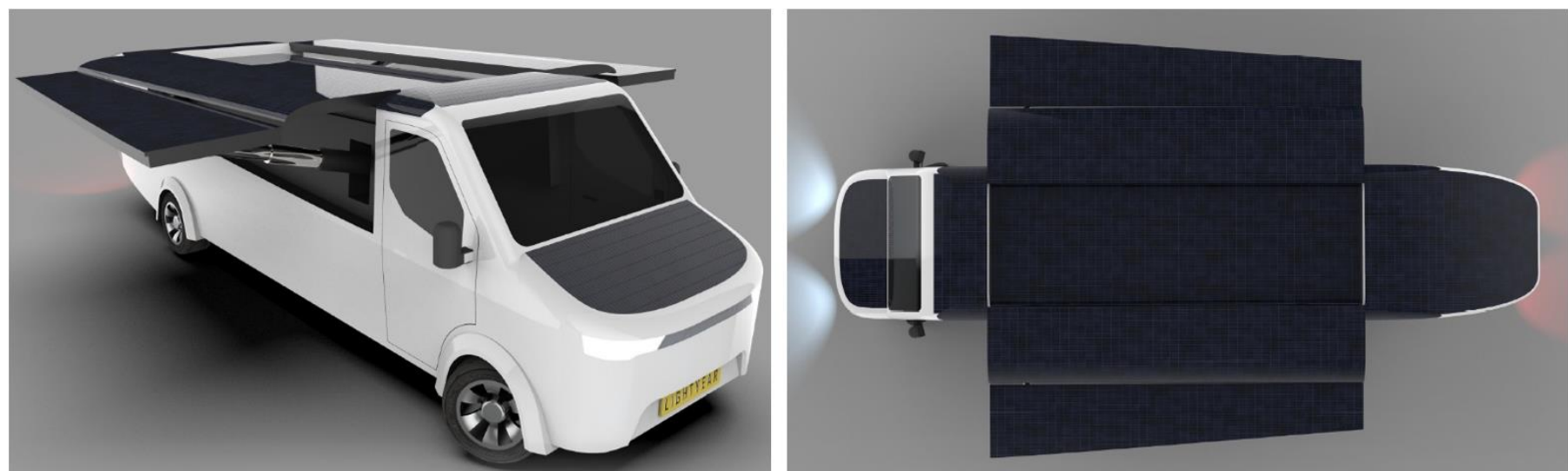

Fig. 4. Lightyear Bus MCU concept by R. den Hertog, S. de Jonge and T. Willems. 


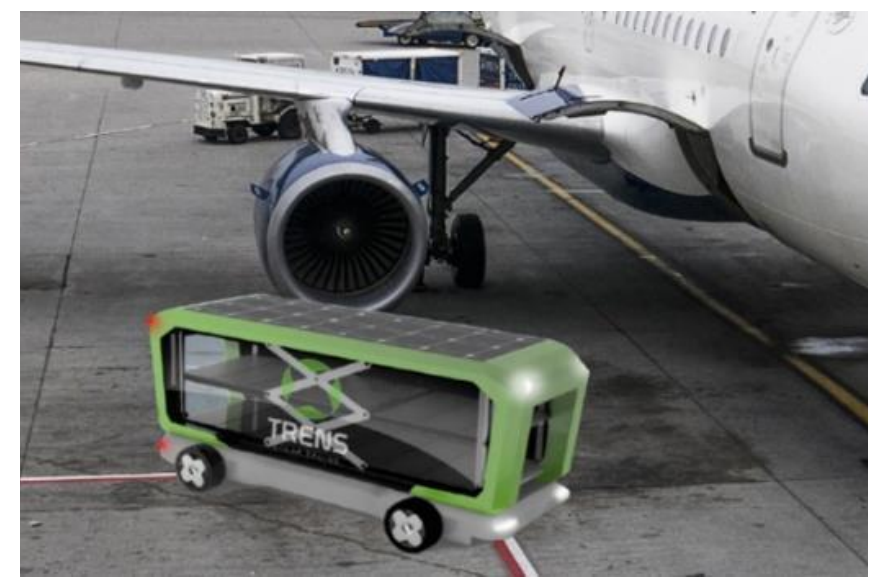

Fig. 5. Solar-powered luggage vehicle concept by J. Liao, N. Pizzigoni and V. Rachmanda.

The charging service proposed by the designers aims to optimise the use of MCUs by allocating a single unit to multiple users; this requires developing a specific algorithm for solving this resource allocation problem. However, even if this is achieved it might prove difficult to persuade several users to move to a single location if some kind of incentive is not provided.

\section{Solar-powered Luggage Vehicle}

In this design, PV cells are integrated to an utility vehicle for loading and unloading luggage in airports. The cells are located in the vehicle roof which is flat-shaped in order to simplify the integration process. In addition to the PV system, the vehicle can also be charged by the existing charging infrastructure which has already been developed for other airport utility vehicles [20]. The luggage vehicle (shown in Fig. 5) is powered by four inwheel motors, with a small battery pack located in the back section of the chassis. Luggage loading and unloading is done through a multi-level system of conveyor belts which maximises the use of space in the vehicle's storage compartment.

The vehicle is intended to operate autonomously, performing a simple sequence of loading and unloading operations and always following a predetermined route between the airplanes and the terminal building. Vehicle operation is programmed through a dedicated user interface which also monitors its performance and allows for manual control when needed.

Design Evaluation - The designers proposed a fully autonomous vehicle design but at there are several barriers for implementing this technology at present. One such barrier involves safety concerns arising from the lack of a driver aboard the vehicle; while remote control through a user interface was suggested as an option it might not be sufficient to mitigate these risks. Subsequent versions of this concept should therefore include driver control or supervision in their design. Another possible issue might involve shading from surrounding objects and the terminal building itself: airport utility vehicles are normally parked on a specific location and if this location is near or inside a building the on-board PV system will not be able to produce electricity when the vehicle is not in use.
Overall, while this concept offers an innovative mobility solution for utility vehicles in airports, these are environments with predictable traffic and shading conditions which can make it difficult to expand this concept beyond this niche application. A possible first step would be designing similar solutions for other types of vehicles found in airports but if some of these design limitations are overcome, it might be eventually possible to develop solutions for other applications such as last-mile delivery in urban locations.

\section{Discussion AND CONCLUSIONS}

A total of eleven design solutions were developed in this study, seven of which focused on charging infrastructure and five on vehicle-integrated PV systems. Designs also included a product-service combination as well as a service-only solution specifically aimed at one of the companies mentioned in Section I. The developed concepts focused on several different vehicle types such as passenger cars, buses and electric bicycles with either public, private or shared ownership.

One of the main design limitations found in this study was the small area available for installing PV systems, particularly for designs which integrated PV cells on vehicles. Extendable arrays were proposed as one of the possible solutions; this is feasible in principle given that vehicles are stationary most of the time but could become problematic in urban environments where there is usually limited space around parked vehicles. In general, vehicle-integrated PV designs focused on larger vehicles such as vans and buses which allowed the vehicle designs to use larger, flatter surfaces in order to provide more surface area for the array and make the PV cells easier to integrate into the vehicle body. Furthermore, the concept for a PV-powered airport utility vehicle shows there might be some interesting niches for VIPV technology beyond passenger transportation.

Designs for PV-powered charging infrastructure, on the other hand, showed how stationary PV systems can be used for powering a wide range of modes of transportation beyond electric passenger cars. The extent to which the energy produced by these systems will meet vehicle demand will vary significantly; PV systems are likely to provide sufficient power for electric bicycles and a significant share of a passenger car's energy requirements but is unlikely to generate enough electricity for charging a bus or a city train. The use of alternative charging technologies such as battery swapping and inductive charging presents an innovative approach to this issue but is difficult to be implemented in practice because of the current limitations of these technologies.

An important aspect of designing these applications is the use of their visual appearance not only for providing an aesthetically pleasing element to the urban environment but also for communicating to users their function and their focus on sustainability. Remarkably, none of the projects considered changing the colour, transparency, texturing or shape of PV cells. This could mean that these features are not as relevant to designers as previously thought and that the current attributes of PV technologies are already perceived to have value from a design perspective. 
Finally, it is important to consider that the presented designs were developed at the conceptual stage only and as such it is difficult to evaluate design aspects such as estimated costs, user acceptance and the required balance of system (BOS) components for each concept. Further development of these concepts is therefore required before their implementation, and should be carried out in combination with urban development projects.

\section{ACKNOWLEDGMENT}

The authors would like to thank all students of Industrial Design Engineering who participated in the 2019 course Sources of Innovation which generated the results shown in this paper. Thanks to Mika Dijkstra, Akshay Suresh, Shrikanth Ramana, Suriya Elango, Uthaiah Parvangada, Gusthavo Ribeiro, Rens den Hertog, Sjoerd de Jonge, Thimo Willems, Jiacheng Liao, Nicola Pizzigoni, Viriega Rachmanda, Jerin Varghese, Omar Martínez, Dan Nguyen, Danny Schmidt, Michel Vos, Alex Reus, Job Schutte, Ellis van Steenis, Stijn Eikenaar, Merel de Smit, Arnt Vollmar, Thomas de Jong, Sam Suidgeest, Auke van der Veen, Joris Kaal, Hanneke Reuvekamp and Léa Texier for allowing us to use their design concepts as part of our research.

\section{REFERENCES}

[1] R. Sims, R. Schaeffer et al., 'Transport. In: Climate Change 2014: Mitigation of Climate Change.' Intergovernmental Panel on Climate Change, 2014.

[2] A. Sierra, T. de Santana, I. MacGill, N. J. Ekins - Daukes, and A. Reinders, 'A feasibility study of solar PV - powered electric cars using an interdisciplinary modeling approach for the electricity balance, $\mathrm{CO}_{2}$ emissions, and economic aspects: The cases of The Netherlands, Norway, Brazil, and Australia', Progress in Photovoltaics: Research and Applications, 2019.

[3] A. Sierra, C. Gercek, and A. Reinders, 'Technical, financial and environmental feasibility analysis of photovoltaic EV charging stations with energy storage in China and the United States', (Unpublished), 2020.

[4] A. Reinders, J. Diehl, and H. Brezet, The power of design: product innovation in sustainable energy technologies. John Wiley \& Sons, 2012

[5] A.H.M.E. Reinders, Designing with Photovoltaics, ISBN: 978-1-31509792-3, Taylor and Francis Group, CRC Press, Boca Raton, USA, 2020.
[6] M. van Geenhuizen, J. Schoonman, and A. Reinders, Diffusion of solar energy use in the built environment supported by new design, Journal of Civil Engineering and Architecture, Vol. 8, No. 2, 53-260, 2014.

[7] T. Gorter, P. Joore, A. Reinders and F. van Houten, Scenario-based simulation of PV boats in an early design stage, 39th IEEE Photovoltaic Specialists Conference, Tampa, 2013.

[8] W. Eggink and A. Reinders, 'Design it with LSCs; an exploration of applications for Luminescent Solar Concentrator PV technologies', in 2017 IEEE 44th Photovoltaic Specialist Conference (PVSC), 2017, pp. 2109-2113.

[9] A. Reinders, M. Wiesenfarth, and R. R. King, 'Conceptual product development with integrated concentrating PV systems - CPV in the built environment from a designer's perspective', in 2013 IEEE 39th Photovoltaic Specialists Conference (PVSC), 2013, pp. 0474-0479.

[10] J. Buijs, 'The Delft Innovation Method A Design Thinker's Guide to Innovation', in DS 71: Proceedings of NordDesign 2012, the 9th NordDesign conference, Aarlborg University, Denmark. 22-24.08. 2012, 2012.

[11] V. Souchkov, 'Breakthrough thinking with TRIZ for business and management: An overview', ICG Training \& Consulting, pp. 3-12, 2007.

[12] J. I. Halman, A. P. Hofer, and W. Van Vuuren, 'Platform - driven development of product families: linking theory with practice' , Journal of product innovation management, vol. 20, no. 2, pp. 149-162, 2003.

[13] W. Eggink and A. Reinders, 'Product integrated PV: the future is design and styling', in 32nd European Photovoltaic Solar Energy Conference and Exhibition, EU PVSEC 2016, 2016, pp. 2818-2821.

[14] U. Obinna, A. Reinders, P. Joore, and L. Wauben, 'A design-driven approach for developing new products for smart grid households', in IEEE PES Innovative Smart Grid Technologies, Europe, 2014, pp. 1-6.

[15] 'Lightyear One: The electric car that charges itself with sunlight', Lightyear, 2019. [Online]. Available: https://lightyear.one/lightyear-one/. [Accessed: 10-Dec-2019].

[16] 'IM Efficiency: Start reducing fuel consumption with green energy', IM Efficiency. [Online]. Available: https://imefficiency.com/index. [Accessed: 20-Jan-2020].

[17] 'Trens Solar Trains', Trens Solar Trains. [Online]. Available: https://www.trens.eu/en. [Accessed: 20-Jan-2020].

[18] B. Zhang. 'Tesla's battery-swapping plan has a mere shadow of the promise it once showed'. Business Insider, 27-Jun-2015. [Online]. Available: https://www.businessinsider.com/teslas-battery-swappingplan-isnt-working-out-2015-6? international $=$ true \& $\mathrm{r}=\mathrm{US} \& \mathrm{IR}=\mathrm{T}$

[19] M. Kane. 'U.S Gets Its First Wireless Fast Charging Bus'. InsideEVs, 01May-2018. [Online]. Available: https://insideevs.com/news/337383/usgets-its-first-wireless-fast-charging-bus/

[20] 'Electric Ground Support Equipment at Airports'. National Renewable Energy Laboratory (NREL), Dec-2017. [Online]. Available: https://afdc.energy.gov/files/u/publication/egse_airports.pdf 